\title{
Discontinuation and comparative effectiveness of dimethyl fumarate and fingolimod in 2 centers
}

Brandi Vollmer, MPH, Daniel Ontaneda, MD, MSc, Anasua Bandyopadhyay, BS, Sam Cohn, MD, Kavita Nair, PhD, Stefan Sillau, PhD, Robert A. Bermel, MD, John R. Corboy, MD, Robert J. Fox, MD, Timothy Vollmer, MD, Jeffrey A. Cohen, MD, Enrique Alvarez, MD, PhD, and Carrie M. Hersh, DO, MSc
Correspondence

Dr. Carrie M. Hersh hershc@ccf.org

Neurology: Clinical Practice August 2018 vol. 8 no. 4 292-301 doi:10.1212/CPJ.0000000000000487

\begin{abstract}
\section{Background}

Dimethyl fumarate (DMF) and fingolimod (FTY) are approved oral disease-modifying therapies for relapsing multiple sclerosis (MS). Observational studies are valuable when randomized clinical trials cannot be done due to ethical or practical reasons. Two-site studies allow investigators to further ascertain external validity of previously examined treatment effect differences. Limited head-to-head 2-site studies exist comparing DMF and FTY.
\end{abstract}

\section{Methods}

Patients prescribed DMF $(\mathrm{n}=737)$ and FTY $(\mathrm{n}=535)$ from 2 academic multiple sclerosis (MS) centers (Cleveland Clinic and University of Colorado) were identified. Discontinuation and disease activity endpoints were assessed using propensity score (PS) weighting. Covariates used in the PS model included demographics and clinical and MRI characteristics.

\section{Results}

PS weighting demonstrated excellent covariate balance. Discontinuation was more common in DMF (44.2\%) compared to FTY (34.8\%) over 24 months (odds ratio [OR] 1.55, 95\% confidence interval $[\mathrm{CI}] 1.21-1.99, p<0.001)$. The leading cause for discontinuation was intolerability for both DMF (56.1\% of DMF discontinuations) and FTY (46.2\% of FTY discontinuations) (OR 1.65, 95\% CI 1.21-2.25, $p=0.002$ ). The proportion of patients with clinical relapses was low for both medications (DMF, 15.1\%; FTY, 13.1\%). There was no difference in the proportion of patients with relapses (OR 1.27, 95\% CI 0.90-1.80, $p=0.174$ ), gadolinium-enhancing lesions (OR 1.42, 95\% CI 0.92-2.20, $p=0.114$ ), or new T2 lesions on brain MRI (OR 1.13, 95\% CI 0.83-1.55, $p=0.433$ ).

\section{Conclusions}

This combined analysis suggests DMF and FTY have similar effectiveness in a large, 2-site clinical population over 24 months. Discontinuation of both DMTs was common and occurred more frequently with DMF, largely driven by intolerability.

Rocky Mountain Multiple Sclerosis Center at Anschutz Medical Campus (BV, KN, SS, JRC, TV, EA), University of Colorado, Denver; Mellen Center for Multiple Sclerosis Treatment and Research (DO, RAB, RJF, JAC), Cleveland Clinic, OH; Emory University (AB), Atlanta, GA; Department of Neurology (SC), Cleveland Clinic, OH; and Lou Ruvo Center for Brain Health (CMH), Cleveland Clinic, Las Vegas, NV.

Funding information and disclosures are provided at the end of the article. Full disclosure form information provided by the authors is available with the full text of this article at Neurology.org/cp.

The Article Processing Charge was funded by Rocky Mountain MS Center at University of Colorado and Cleveland Clinic Lou Ruvo Center for Brain Health.

This is an open access article distributed under the terms of the Creative Commons Attribution-NonCommercial-NoDerivatives License 4.0 (CC BY-NC-ND), which permits downloading and sharing the work provided it is properly cited. The work cannot be changed in any way or used commercially without permission from the journal. 
In recent years, there has been a dramatic rise in available disease-modifying therapies (DMTs) approved for the treatment of multiple sclerosis (MS), including the introduction of oral medications such as dimethyl fumarate (DMF) and fingolimod (FTY). DMF (240 mg, 1 tablet twice daily) was approved by the US Food and Drug Administration (FDA) and European Medicines Agency in 2013. ${ }^{1,2}$ FTY (0.5 mg, 1 tablet once daily), a sphingosphine-1 phosphate receptor modulator, was approved by the FDA in 2010 and European Medicines Agency in 2011. ${ }^{3-5}$

Phase 3 randomized controlled trials (RCTs) showed similar reductions in the annualized relapse rate (ARR) of 44\%-53\% for DMF and $48 \%-54 \%$ for FTY, when compared against placebo. ${ }^{1,2,4,5}$ However, DMF and FTY tolerability profiles had prominent differences. By 24 months, $12 \%-16 \%$ and $5.6 \%-8.5 \%$ of DMF and FTY patients discontinued treatment in the RCTs due to adverse events (AEs), respectively. ${ }^{1,2,4,5}$ Common DMF AEs included flushing and gastrointestinal issues, such as nausea, diarrhea, and vomiting, which were most prominent during the first 1-2 months of treatment. ${ }^{1,2}$ Common FTY AEs included mild to moderate upper respiratory tract infections, headaches, and back pain. Rare but more important risks included lymphopenia and cardiovascular AEs, fatal herpesvirus infections, and macular edema. ${ }^{5,6}$ Rare cases of progressive multifocal leukoencephalopathy have been reported for both DMF and FTY. ${ }^{7-10}$

No RCT directly comparing DMF and FTY has been conducted. Previous single-site, observational studies in a realworld setting demonstrated similar effectiveness outcomes and an increased likelihood of discontinuing DMF by 24 months compared to FTY. ${ }^{11-13}$ Two-site studies may allow investigators to ascertain external validity of previously examined treatment effect differences. Therefore, we aimed to address this knowledge gap by comparing discontinuation and effectiveness of DMF and FTY over 24 months in patients treated at 2 large academic MS centers. Propensity score (PS) analysis was used to reduce confounding and certain biases. ${ }^{14}$

\section{Methods}

\section{Standard protocol approvals, registrations, and patient consents}

This study was approved by institutional review boards at the Cleveland Clinic and University of Colorado. Informed consent was waived for this study.

\section{Patient population}

We conducted a retrospective observational study of patients with MS followed at the Cleveland Clinic Mellen Center or the Rocky Mountain MS Center at the University of Colorado (RMMSC at CU) treated either with DMF or FTY with available 24-month follow-up data. Patients treated at Cleveland Clinic were selected from those starting DMF or
Relapsing and progressive MS were included in this study to demonstrate the real-world experience of DMT use.

FTY within 1 year of respective FDA approval. Patients treated at RMMSC at CU were selected from those starting DMF or FTY prior to October 2013. Each center included a sample size reflective of individual treatment arms in the respective phase 3 trials. ${ }^{1,2,4,5}$ Relapsing and progressive MS were included in this study to demonstrate the real-world experience of DMT use. Subgroup analyses were also conducted for patients with relapsing-remitting MS (RRMS) and for groups stratified by age $(<40$ years old $/ \geq 40$ years old), sex (male/female), drug line use (first-line/non-firstline), presence of gadolinium-enhancing (GdE) lesions on baseline brain MRI (present/not present), baseline MRI lesion burden (mild/moderate to severe), and patients who switched directly from natalizumab to either DMF or FTY.

\section{Data collection}

Patients who met inclusion criteria were identified, and a retrospective chart review of the electronic medical record (EMR) was completed at each center to collect baseline and outcomes data 24 months after DMT initiation. Baseline covariates were collected from 12 months prior to respective treatment exposure.

Follow-up postbaseline (e.g., MRI frequency/protocols and visits) was similar between groups. Clinical relapses, defined as new or worsening MS symptoms lasting greater than 24 hours without a coexistent illness or fever, were verified by the treating clinicians and documented in the EMR. Number of new T2 brain lesions and semiquantitative assessment of overall lesion burden were determined manually by C.M.H. at Cleveland Clinic, B.V. at RMMSC at CU, and neuroradiologists at each institution.

Cleveland Clinic data were stored using Redcap software and stored on a secure Cleveland Clinic server. RMMSC at CU data were encrypted and stored on a secure server using Excel. The data from each center were de-identified and merged into a single Excel database.

\section{Outcome measures}

The primary outcome was the proportion of patients who discontinued DMF or FTY by 24 months. Secondary outcomes included reason for discontinuation (categorized as intolerance or disease activity), time to discontinuation, proportion with clinical relapses, proportion with brain MRI GdE lesions, proportion with new brain MRI T2 lesions, proportion with brain MRI activity (defined as new T2 or GdE lesions), and proportion with absence of disease activity 
(defined as freedom from clinical relapses and MRI activity). All effectiveness outcomes, including clinical relapse and MRI activity, were on-treatment measures.

\section{Statistical analysis}

De-identified data were imported into $\mathrm{R}$ version 3.4.2 for analysis. ${ }^{15}$ We used the same approach as in our previous 12 and 24-month studies to account for missingness patterns in the PS. ${ }^{12,16}$ Two-tailed $p$ values $<0.05$ were considered statistically significant. Differences between groups for unadjusted estimates were assessed using $t$ tests for continuous data, $\chi^{2}$ tests for categorical data, and Cox proportional hazards models and Kaplan-Meier survival analyses for survival outcomes. ${ }^{12,16}$ Adjusted analyses were conducted using PS methods.

The PS model was built as a logistic regression to calculate the likelihood of initiating DMF, as compared to FTY, using preselected demographics and baseline clinical and MRI characteristics (figure e-1, links.lww.com/CPJ/A33). A PS was derived for each patient and used in average treatment effect on the treated (ATT) weighting to identify patients in the DMF and FTY groups who were comparable at baseline except for treatment. All covariates included were missing in fewer than $10 \%$ of patients.

Similar to our previous single-center 24-month study, we compared the standardized differences in covariates before and after propensity adjustment to determine the strength of our weighting method. ${ }^{12}$ Excellent covariate balance was defined as an absolute standardized difference $<10 \%$ on the covariate means across the 2 DMTs. Treatment groups were compared with conditional logistic regression to obtain odds ratio (OR) estimates for binary outcomes after ATT weighting. Survival data were estimated by stratified Cox regression and Kaplan-Meier survival curves. ORs and hazards ratios (HRs) refer to patients treated with DMF compared to those treated with FTY. ${ }^{12}$

\section{Data Availability}

Anonymized data can be shared at the request of qualified investigators for purposes of replicating procedures and results.

\section{Results}

\section{Baseline characteristics}

Figure 1 shows the overall study flow. A total of 1,272 patients were included. Baseline characteristics are presented in table 1. Differences were observed in the following covariates: age, race, type of MS, last DMT prior to DMF or FTY, and baseline MRI lesion burden (see table 1 for details).

\section{Propensity model}

The propensity model was created using covariates included in table 1. Missing data among covariates in the PS model did not meaningfully change overall covariate balance after PS analysis. Prior to propensity adjustment, the treatment

Figure 1 Study flow diagram

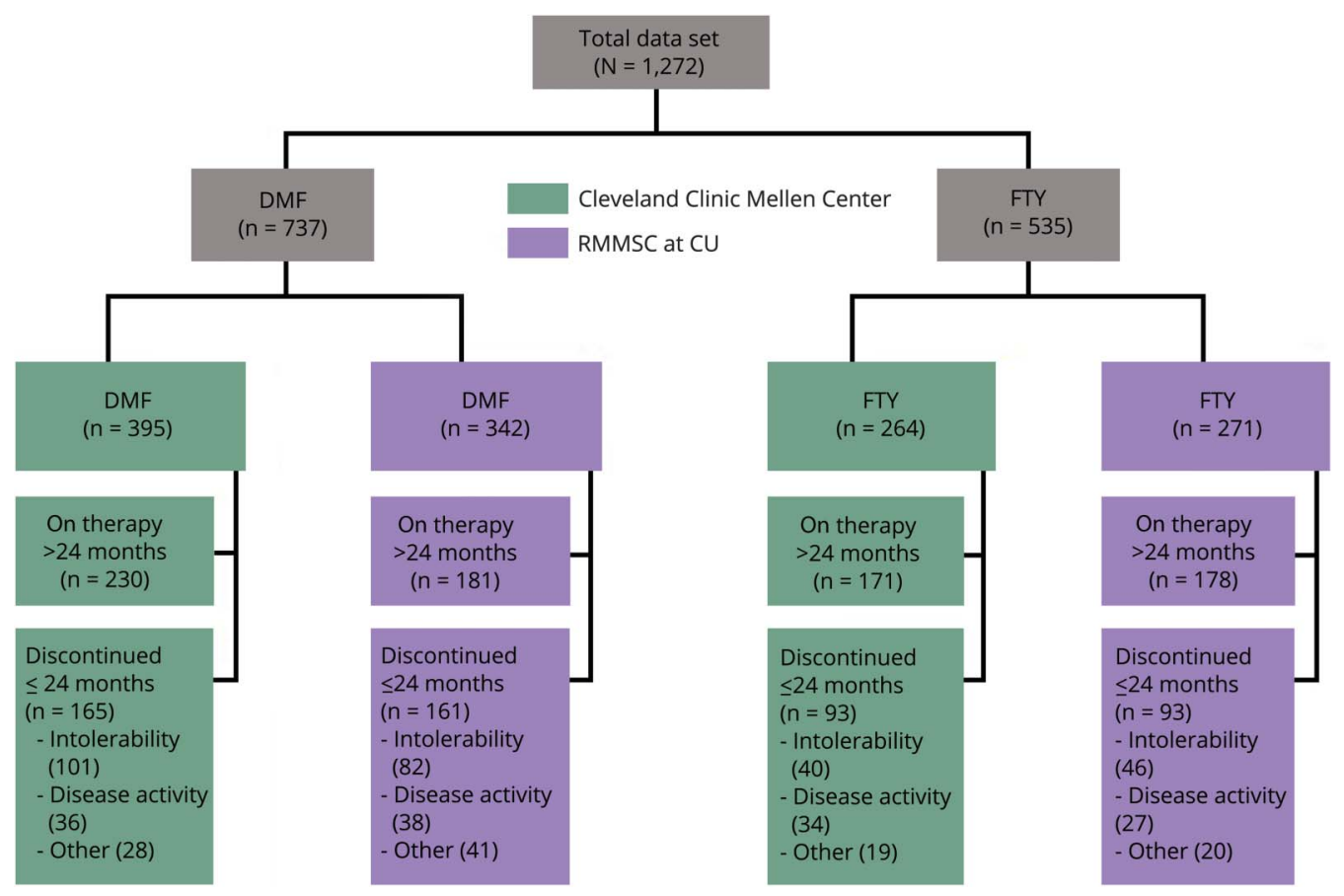

Discontinuations due to other include pregnancy, insurance issues, patient preference, and loss to follow-up. DMF = dimethyl fumarate; FTY = fingolimod; RMMSC at CU = Rocky Mountain Multiple Sclerosis Center at the University of Colorado. 
Table 1 Baseline characteristics of study population

\begin{tabular}{|c|c|c|c|c|c|}
\hline & \multicolumn{2}{|l|}{ DMF $(n=737)$} & \multicolumn{2}{|l|}{ FTY $(n=535)$} & \multirow[b]{2}{*}{$p$ Value $^{a}$} \\
\hline & No. or mean & $\%$ Or SD & No. or mean & $\%$ Or SD & \\
\hline Age, y & 46.4 & 11.6 & 43.3 & 10.4 & $<0.001^{a}$ \\
\hline Sex, female & 516 & 70.0 & 382 & 71.4 & N/S \\
\hline Race & & & & & $0.005^{\mathrm{a}}$ \\
\hline White & 614 & 87.2 & 476 & 93.0 & \\
\hline Black & 59 & 8.4 & 22 & 4.3 & \\
\hline Other & 31 & 4.4 & 14 & 2.7 & \\
\hline Not reported & 33 & 4.5 & 23 & 4.3 & \\
\hline Disease duration, y & 13.4 & 9.3 & 13.3 & 8.6 & N/S \\
\hline Relapsing-remitting MS & 558 & 75.7 & 459 & 85.8 & $<0.001^{a}$ \\
\hline Secondary progressive MS & 119 & 16.1 & 53 & 9.9 & $0.010^{\mathrm{a}}$ \\
\hline Primary progressive MS & 60 & 8.1 & 23 & 4.3 & $0.030^{\mathrm{a}}$ \\
\hline Last therapy prior to DMF/FTY & & & & & $<0.001^{a}$ \\
\hline None $^{b}$ & 282 & 38.3 & 159 & 29.7 & \\
\hline Interferon & 127 & 17.2 & 103 & 19.3 & \\
\hline Glatiramer & 198 & 26.9 & 110 & 20.6 & \\
\hline Natalizumab & 91 & 12.3 & 139 & 26.0 & \\
\hline Immunosuppression & 26 & 3.5 & 15 & 2.8 & \\
\hline Other & 13 & 1.8 & 9 & 1.7 & \\
\hline Baseline MRI available & 677 & 91.9 & 482 & 90.1 & N/S \\
\hline Disease burden on baseline MRI & & & & & $<0.001^{a}$ \\
\hline Mild & 374 & 55.2 & 193 & 40.0 & \\
\hline Moderate & 245 & 36.2 & 210 & 43.6 & \\
\hline Severe & 58 & 8.6 & 79 & 16.4 & \\
\hline GdE lesions on baseline MRI & 133 & 19.4 & 115 & 23.5 & N/S \\
\hline
\end{tabular}

Abbreviations: $\mathrm{DMF}=$ dimethyl fumarate; DMT = disease-modifying therapy; FTY = fingolimod; GdE = gadolinium-enhancing; MS = multiple sclerosis. In our study, DMF patients were more likely to be older, have milder baseline lesion burden on brain MRI, and have been on no previous therapy. FTY patients were more likely to be white, have relapsing-remitting MS, and previously been on natalizumab.

a Statistically significant $p$ value $(a=0.05)$.

${ }^{\mathrm{b}}$ Patients who were remote switchers (>3 months since last DMT) or first-line users.

groups were not well-balanced (absolute value of the standardized difference of the linear PS, comparing DMF to FTY $=51.8 \%)$. We effectively achieved well-balanced groups through ATT weighting with no covariates having an absolute standardized difference greater than $10 \%$ (figure e-1, links.lww.com/CPJ/A33). PS weighting also produced a comparable linear PS distribution with a standardized difference of $4.3 \%$, well within the $50 \%$ standard proposed by Rubin. ${ }^{17}$

\section{Outcomes for overall population}

Tables 2 and 3 present a summary of unadjusted and propensity-adjusted outcome estimates using ATT weighting. Figure e-2 (links.lww.com/CPJ/A33) demonstrates Kaplan-Meier plots for DMT discontinuation for any reason, due to intolerability, and due to MRI activity through 24 months. Discontinuation was more common in DMF patients $(\mathrm{n}=326,44.2 \%)$ compared to FTY patients $(\mathrm{n}=186,34.8 \%)$ over 24 months (OR $1.55,95 \%$ confidence interval [CI] 1.21-1.99, $p<0.001)$ and occurred earlier in DMF patients (HR 1.48, 95\% CI $1.25-1.74, p<0.001)$, as confirmed via Kaplan-Meier survival curves (figure e-2a). Intolerability was the most common reason for discontinuation in both groups (DMF $\mathrm{n}=183,24.8 \%$; FTY $\mathrm{n}=86,16.1 \%$ ), though DMF patients were more likely to discontinue therapy due to 
Based on our results, intolerability appeared to be the driving force for the observed difference in discontinuation between DMF and FTY.

intolerability compared to FTY patients (OR 1.65, 95\% CI $1.21-2.25, p=0.002)$. The primary reason for discontinuation due to intolerability was gastrointestinal side effects with DMF (57.9\%) and headaches with FTY (14.0\%). There was no difference between DMF and FTY for discontinuation due to disease activity (DMF $\mathrm{n}=74$, 10.0\%; FTY n =61, 11.4\%; OR 1.03, 95\% CI 0.69-1.53, $p=$ $0.892)$.

PS weighting analyses demonstrated no differences between DMF and FTY for any effectiveness outcome, including proportion with clinical relapses (OR 1.27, 95\% CI $0.90-1.80, p=0.174$ ), brain MRI activity (OR 1.08, 95\% CI $0.80-1.46, p=0.595$ ), new T2 lesions (OR 1.13, 95\% CI
$0.83-1.55, p=0.433$ ), and new GdE lesions (OR 1.42, 95\% CI 0.92-2.20, $p=0.114$ ). By 24 months, 325 DMF patients (61.2\%) demonstrated absence of disease activity vs 282 FTY patients (63.4\%) (OR 0.84, 95\% CI 0.70-1.27, $p=$ $0.268)$.

\section{Subgroup analyses}

Figure 2 and tables e-1 through e-14 (links.lww.com/CPJ/ A34) present a summary of unadjusted outcome estimates and propensity-adjusted outcome estimates using ATT weighting for patients with RRMS and groups stratified by age, sex, drug line use, presence of GdE lesions at baseline, baseline MRI lesion burden, and patients who switched from natalizumab. Patients with RRMS demonstrated results consistent with the overall group, with the exception of DMF demonstrating increased odds of GdE lesions compared to FTY (OR 1.62, 95\% CI 1.02-2.58). After PS adjustment, older patients were more likely to discontinue DMF compared to FTY (OR 1.43, 95\% CI 1.04-1.95), whereas younger patients were equally likely to discontinue either therapy (OR 1.48, 95\% CI 0.95-2.31). Natalizumab switchers were equally likely to remain on FTY or DMF (OR 1.41, 95\% CI 0.79-2.54). Women had higher likelihood of DMF discontinuation due to intolerability compared to FTY-treated patients (OR 2.17, 95\% CI 1.50-3.13), which was not seen in men. Men

Table 2 Unadjusted outcomes at 24-month follow-up

\begin{tabular}{|c|c|c|c|c|c|}
\hline & \multicolumn{2}{|l|}{ DMF $(n=737)$} & \multicolumn{2}{|l|}{ FTY $(n=535)$} & \multirow[b]{2}{*}{$p$ Value $^{a}$} \\
\hline & No. or mean & $\%$ Or SD & No. or mean & $\%$ Or SD & \\
\hline Discontinued drug $\leq 24$ months & 326 & 44.2 & 186 & 34.8 & $0.001^{a}$ \\
\hline Disease activity & 74 & 10.0 & 61 & 11.4 & 0.493 \\
\hline Intolerance & 183 & 24.8 & 86 & 16.1 & $<0.001^{a}$ \\
\hline Mean time to discontinuation, mo & 3.73 & 3.46 & 6.55 & 4.40 & $<0.001^{\mathrm{a}}$ \\
\hline Clinical relapse $\leq 24$ months & 111 & 15.1 & 70 & 13.1 & 0.350 \\
\hline Relapses per patient (mean) & 0.15 & 0.36 & 0.13 & 0.34 & 0.310 \\
\hline MRI available $\leq 24$ months on DMT & 533 & 72.3 & 446 & 83.4 & 0.095 \\
\hline $\begin{array}{l}\text { Disease activity on MRI } \leq 24 \text { months on } \\
\text { DMT }\end{array}$ & 154 & 28.9 & 127 & 28.5 & 0.945 \\
\hline GdE lesions & 62 & 11.7 & 50 & 11.2 & 0.918 \\
\hline New T2 lesions & 140 & 26.3 & 107 & 24.0 & 0.460 \\
\hline MRI available 12-24 months on DMT & 282 & 38.3 & 246 & 45.9 & 0.108 \\
\hline $\begin{array}{l}\text { Disease activity on MRI 12-24 months on } \\
\text { DMT }\end{array}$ & 48 & 17.0 & 41 & 16.7 & 1.000 \\
\hline GdE lesions & 23 & 8.1 & 14 & 5.7 & 0.362 \\
\hline New T2 lesions & 40 & 14.1 & 36 & 14.8 & 0.938 \\
\hline Absence of disease activity ${ }^{b}$ & 325 & 61.2 & 282 & 63.4 & 0.268 \\
\hline
\end{tabular}

Abbreviations: DMF = dimethyl fumarate; DMT = disease-modifying therapy; FTY = fingolimod; GdE = gadolinium-enhancing.

b Proportion of patients free of clinical relapses, GdE lesions, and new T2 lesions calculated from those with complete data available (DMF $\mathrm{n}=531$, FTY $\mathrm{n}=445$ ). 
Table 3 Unadjusted and adjusted endpoints of DMF compared to FTY at 24 months

\begin{tabular}{|c|c|c|c|c|c|c|}
\hline \multirow[b]{2}{*}{ Study endpoints } & \multicolumn{3}{|l|}{ Unadjusted } & \multicolumn{3}{|c|}{ Propensity adjusted } \\
\hline & $\begin{array}{l}\text { Odds or } \\
\text { hazards ratio }\end{array}$ & $95 \% \mathrm{Cl}$ & $p$ Value $^{a}$ & $\begin{array}{l}\text { Odds or } \\
\text { hazards ratio }\end{array}$ & $95 \% \mathrm{Cl}$ & $p$ Value $^{a}$ \\
\hline DMT discontinuation & 1.49 & $1.18-1.87$ & $<0.001^{a}$ & 1.55 & $1.21-1.99$ & $<0.001^{a}$ \\
\hline Disease activity & 0.87 & $0.61-1.24$ & 0.461 & 1.03 & $0.69-1.53$ & 0.892 \\
\hline Intolerability & 1.72 & $1.30-2.29$ & $<0.001^{a}$ & 1.65 & $1.21-2.25$ & $0.002^{a}$ \\
\hline Time to discontinuation & 1.45 & $1.19-1.71$ & $<0.001^{a}$ & 1.48 & $1.25-1.74$ & $<0.001^{a}$ \\
\hline Clinical relapse & 1.18 & $0.86-1.63$ & 0.330 & 1.27 & $0.90-1.80$ & 0.174 \\
\hline Brain MRI activity $\leq 24$ months & 1.02 & $0.77-1.35$ & 0.943 & 1.08 & $0.80-1.46$ & 0.595 \\
\hline GdE lesions & 1.04 & $0.70-1.55$ & 0.586 & 1.42 & $0.92-2.20$ & 0.114 \\
\hline New T2 lesions & 1.13 & $0.84-1.51$ & 0.460 & 1.13 & $0.83-1.55$ & 0.433 \\
\hline Brain MRI activity $12-24$ months & 1.02 & $0.64-1.61$ & 1.000 & 1.00 & $0.62-1.63$ & 0.986 \\
\hline GdE lesions & 1.46 & $0.74-2.90$ & 0.309 & 1.94 & $0.92-4.08$ & 0.081 \\
\hline New T2 lesions & 0.95 & $0.58-1.55$ & 0.901 & 0.91 & $0.54-1.52$ & 0.715 \\
\hline Absence of disease activity & 0.91 & $0.85-1.42$ & 0.508 & 0.84 & $0.70-1.27$ & 0.268 \\
\hline
\end{tabular}

Abbreviations: $\mathrm{Cl}$ = confidence interval; DMF = dimethyl fumarate; DMT = disease-modifying therapy; FTY = fingolimod; GdE = gadolinium-enhancing.

a Statistically significant $p$ value $(a=0.05)$

${ }^{b}$ Proportion of patients free of clinical relapses, GdE lesions, and new T2 lesions calculated from those with complete data available.

treated with DMF vs FTY had higher likelihood of GdE lesions (OR 2.78, 95\% CI 1.32-5.82), though relapse rates and new T2 lesions were comparable. DMF first-line users had a higher likelihood of clinical relapses (OR 1.56, 95\% CI 1.08-3.57) compared to FTY first-line users. No other subgroup analyses demonstrated differences in effectiveness outcomes.

\section{Discussion}

We investigated the discontinuation and comparative effectiveness of DMF and FTY over 24 months in a large population from 2 academic MS centers with a sample size exceeding that in most MS phase 3 RCTs. Consistent with our previous 24-month single-center studies, DMF demonstrated higher odds of discontinuation compared to FTY. ${ }^{11,12}$ Further, we observed a greater proportion of discontinuations for both DMF and FTY than what was observed in respective phase 3 RCTs (DMF: $44.2 \%$ vs $30 \%-31 \%$ in phase 3 RCTs; FTY: $34.8 \%$ vs $18.8 \%-32 \%$ in phase 3 RCTs). ${ }^{1,2,4,5}$ This difference highlights the importance of conducting observational studies, for patients in a real-world setting may differ in motivation to remain on treatment compared to those in a highly regimented RCT. For instance, in clinical practice we anticipate that the provider and patient may be less inclined to continue a DMT with AEs or disease activity when other treatment options are available. In addition, our study had broader inclusion criteria, allowing us to investigate effects more representative of the general population treated in clinical practice, including use in progressive disease and older patients.

Based on our results, intolerability appeared to be the driving force for the observed difference in discontinuation between DMF and FTY. Discontinuation due to intolerability occurred earlier with DMF during the first 6 months of treatment and then began to decrease in rate, appearing more similar to FTY, which is reassuring for DMT persistence in longer-term disease management. This finding was consistent with that of clinical trials that demonstrated gastrointestinal AEs to be common in the first 60 days of DMF treatment. ${ }^{1,2}$ Discontinuation due to disease activity was comparable for DMF and FTY in the overall group.

Similar to our single-center 24-month investigations, DMF and FTY demonstrated comparable clinical and radiologic effectiveness, with low proportions of patients who experienced a clinical relapse and MRI activity. These results were also consistent with previous PS-adjusted analyses indirectly comparing DMF and FTY patients from the pivotal phase 3 RCTs and directly comparing DMF and FTY in a large claims database analysis. Both investigations found no differences in efficacy via ARR. ${ }^{18,19}$ In addition, we observed a lower proportion of patients who experienced a relapse over 2 years while on treatment in the entire group (DMF $=15.1 \%$, FTY $=13.1 \%)$ and RRMS subgroup $(\mathrm{DMF}=16.3 \%, \mathrm{FTY}=$ $13.5 \%)$ compared to phase 3 RCTs $(\mathrm{DMF}=27 \%-29 \%$, FTY $=28.5 \%-29.6 \%$ ), which is reassuring for confirming treatment effectiveness in real-world practice. The observed difference was likely, in part, reflective of the inclusion of older 
Figure 2 Forest plots for subgroup analyses

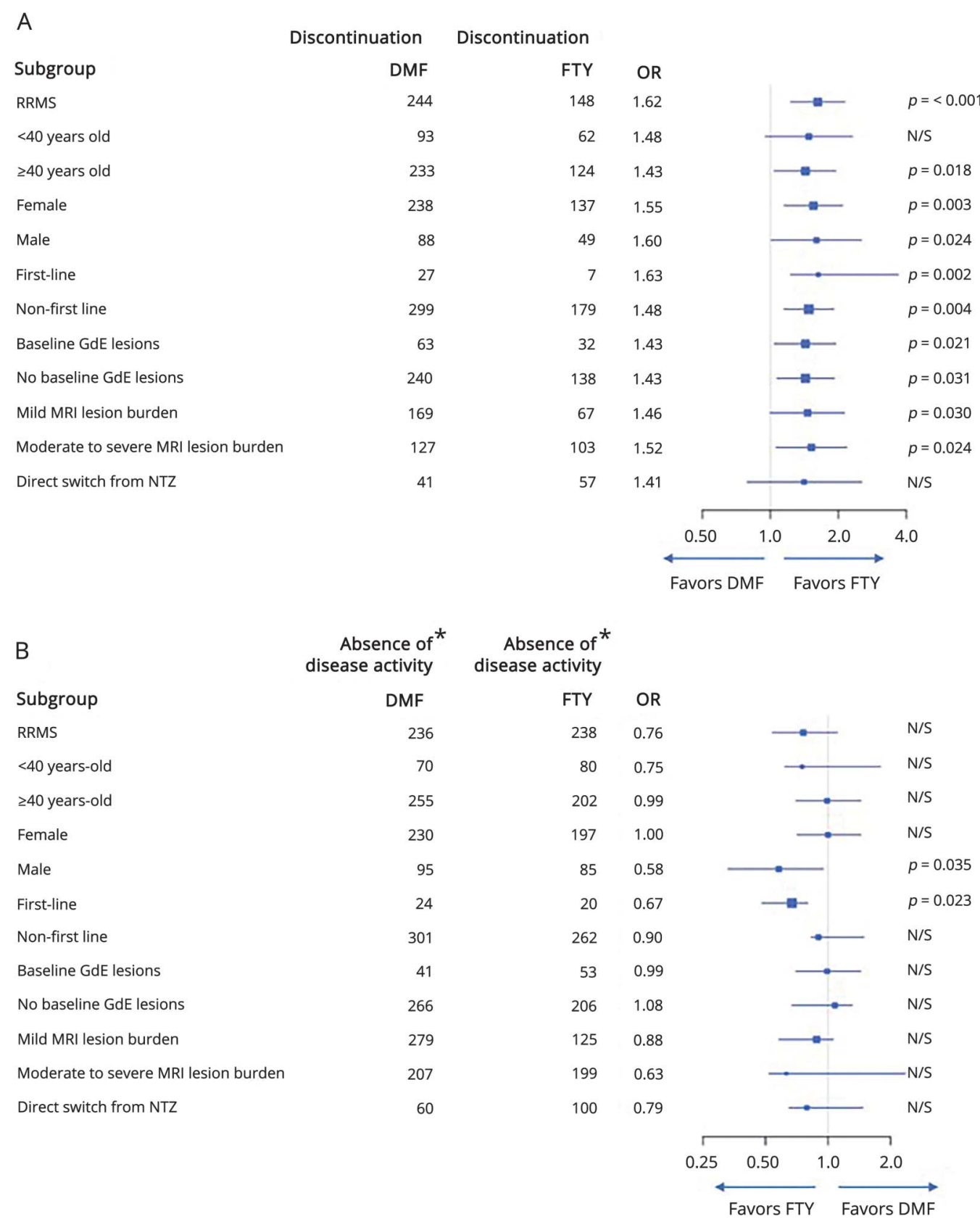

*Free of clinical relapses, GdE lesions, and new T2 lesions calculated from those with complete data available. (A) Forest plot demonstrates discontinuation $\leq 24$-month follow-up for subgroups. (B) Forest plot demonstrates absence of disease activity at 24-month follow-up for subgroups. DMF = dimethyl fumarate; FTY = fingolimod; OR = odds ratio; RRMS = relapsing-remitting multiple sclerosis; GdE = gadolinium-enhancing lesion; NTZ natalizumab; = N/S = nonsignificant.

patients in our study with both active and inactive baseline disease activity, whereas RCTs were restricted to younger patients with recent disease activity. ${ }^{20,21}$

As the number of highly effective treatments for MS grows, the treat-to-target outcome measure of no evidence of disease activity (NEDA), defined as no relapses, disability progression, or MRI activity (new or enlarging T2 lesions or GdE lesions), is increasingly utilized in MS studies. ${ }^{22}$ In our investigation, we measured absence of disease activity (clinical relapses and MRI activity) to explore freedom from inflammatory disease. ${ }^{12}$ Due to the retrospective nature of our study, measures of disability were not available consistently and, therefore, were not included in our definition of absence of disease activity outcome. We found no differences between DMF and FTY. This finding is contradictory to an indirect comparison model of phase 3 RCT data, conducted by Nixon et al., ${ }^{23}$ which demonstrated a reduced probability of achieving NEDA in DMF patients compared to those treated with FTY. We suspect that the reasons for these differences were multifactorial: different study populations (real-world observational data vs clinical trial data), 
types of comparisons (direct vs indirect), and different NEDA/absence of disease activity definitions. ${ }^{23}$

Previous analyses of phase 3 RCT studies demonstrated consistent efficacy for DMF and FTY compared to placebo over a broad range of patient subgroups. ${ }^{24-26}$ The majority of our DMF vs FTY subgroup analyses demonstrated comparable effectiveness across multiple clinical and radiographic outcomes, similar to our overall group. Exceptions were patients with RRMS, first-line users, and men after PS adjustment. For RMMS, DMF patients demonstrated greater likelihood of new GdE lesions than FTY patients; however, the magnitude of this difference was likely small, as the overall MRI activity and absence of disease activity endpoints were comparable. DMF first-line users demonstrated a greater likelihood of clinical relapses and lower likelihood of absence of disease activity, suggestive of reduced effectiveness in this group. In addition, the DMF male population demonstrated a greater likelihood of new GdE lesions. This finding among men observed in our study was consistent with previous literature investigating DMF compared to placebo. In a phase $2 \mathrm{RCT}$ for DMF, the reduction in the number of new GdE lesions remained significant in all subgroups except for male patients. ${ }^{27}$ However, this study investigated DMF at $240 \mathrm{mg} 3$ times daily rather than the approved twice daily formulation, and insignificant results were attributed to small sample sizes and low disease activity at baseline in men. ${ }^{27}$ In addition, while our data suggested improved effectiveness in first-line users and men treated with FTY based on a few endpoints, these were limited either by small sample sizes or wide CIs and warrant cautious interpretation.

Interestingly, women discontinued DMF more due to intolerability, which was not seen in men. Previous research demonstrated that female patients were more likely to discontinue DMTs, ${ }^{28}$ which was supported by our unadjusted results in table e-3 (links.lww.com/CPJ/A34), demonstrating slightly higher proportions of DMT discontinuation in women compared to men. The observed sex differences in DMT discontinuation may be clinically relevant and warrant further investigation.

Although our PS model improved baseline covariate balance, we recognize the possibility for residual and hidden biases that available covariates do not address. However, our PS methods included important baseline characteristics that are incorporated into the shared decision-making model of choosing a DMT in clinical practice. The inclusion of subgroup PS analyses provided additional information on distinct groups that allowed us to confirm treatment effects, including analysis of a separate RRMS subgroup to ascertain treatment effects in a population of patients similar to those in phase 3 RCTs. Furthermore, this study included data from 2 academic MS centers, allowing us to ascertain external validity of previously examined treatment effect differences.
Our study has other limitations, owing to the assumptions inherent in observational studies, even after the application of PS adjustment. Certain challenges, such as balancing patients treated with natalizumab vs those with early disease, are inherent aspects of observational studies regardless of PS analysis. As such, separate subgroup analyses comparing natalizumab switchers were conducted to account for this limitation. Further, differences in counseling practices between providers and DMT administration in patients with lower baseline disease activity who may have lower tolerance for AEs may have contributed to the differences in discontinuation between DMF and FTY, which cannot be accounted for through PS adjustment. While our retrospective chart review design allowed for the inclusion of clinical and MRI data not available through other sources, such as claims data, we were limited by previous clinicianreported and missing data that are inherent aspects of observational studies. As a result, we were unable to clarify inconsistencies in the patient chart if they arose. Particularly when inconsistencies were observed, the investigators used data from the chart note closest in date to the event of interest in order to reduce recall bias. In addition, while inclusion of older and progressive patients is representative of the general population treated in clinical practice, the low inflammatory profiles of these patients may have obscured treatment effect differences. Reassuringly, the majority of effectiveness outcomes in the RRMS subgroup were also comparable. Finally, this study included data from 2 large academic MS centers, which may lessen generalizability to smaller community-based practices with different methods of treatment counseling.

These 2-center results confirm previous single-center 24month studies demonstrating increased odds of DMF discontinuation compared to FTY, largely driven by intolerability. Reassuringly, our data substantiate DMT persistence in longer-term disease management for both DMF and FTY. Similar to respective phase 3 trial data and other observational studies, our results further confirm comparable clinical and radiographic effectiveness profiles of these DMTs in clinical practice.

\section{Author contributions}

B. Vollmer drafted and revised the manuscript and contributed to the study design, acquisition, and interpretation of the data. D. Ontaneda contributed to the study concept and design, interpretation of the data, and revision of the manuscript. A. Bandyopadhyay contributed to the data acquisition. S. Cohn contributed to the data acquisition. K. Nair contributed to the study concept and design, data interpretation, and revision of the manuscript. S. Sillau contributed to the analysis and interpretation of the data. R.A. Bermel contributed to the study concept. J.R. Corboy contributed to the study concept and revision of the manuscript. R.J. Fox contributed to the study concept. T. Vollmer contributed to the study concept and revision of the manuscript. J.A. Cohen contributed to the study concept and revision of the 
manuscript. E. Alvarez contributed to the study concept and design, interpretation of the data, and revision of the manuscript. C.M. Hersh contributed to the study concept, acquisition, analysis, and interpretation of the data, and drafted and revised the manuscript.

\section{Acknowledgment}

The authors thank the patients and clinicians at the Cleveland Clinic Mellen Center and Rocky Mountain Multiple Sclerosis Clinic at the University of Colorado who helped formulate and implement the fingolimod and dimethyl fumarate treatment protocols.

\section{Study funding}

No targeted funding reported.

\section{Disclosure}

B. Vollmer reports no disclosures. D. Ontaneda serves on scientific advisory boards for Genzyme, Genentech, and Biogen Idec; serves as a consultant for Biogen Idec, Genzyme, Genentech, Merck, and the France Foundation; and receives research support from Genzyme, Novartis, Genentech, NIH, NMSS, PCORI, and Race to Erase Foundation. A. Bandyopadhyay and S. Cohn report no disclosures. K. Nair has received funding for travel or speaker honoraria from Sanofi; serves as a consultant for Astellas; serves on the speakers' bureau for AbbVie; and receives research support from Novartis, Biogen, and Genentech. S. Sillau receives research support from University of Colorado. R.A. Bermel serves on scientific advisory boards for Novartis and Genentech; serves as a consultant for Biogen, Genzyme, Genentech, and Novartis; and receives research support from Novartis, Biogen, and Genentech. J.R. Corboy has received speaker honoraria from the Rocky Mountain MS Center, ECTRIMS, and PRIME CME; serves as Editor of Neurology: Clinical Practice; is PI on clinical trials funded by Novartis, Biogen, Med Day, PCORI, and NMSS; receives research support from Novartis, Med Day, Biogen, PCORI, and National MS Society; and has participated in medico-legal cases. R.J. Fox serves on scientific advisory boards for Actelion, Biogen Idec, and Novartis; serves on the editorial boards of Neurology and Multiple Sclerosis Journal; receives publishing royalties for Multiple Sclerosis and Related Disorders (Demos Medical, 2013); serves as a consultant for Actelion, Biogen, EMD Serono, Genentech, Novartis, and Teva; and receives research support from Novartis, Biogen, National MS Society, NIH, and Consortium of MS Centers. T. Vollmer serves on scientific advisory boards/as a consultant for Academic CME, Alcimed, Anthem Blue Cross, Genentech/Roche, Biogen IDEC, Novartis, CellGene, Seono, Epigene, Rocky Mountain MS Center, GLG Consulting, Ohio Health, TG Therapeutics, Topaz Therapeutics, Dleara Lawyers, and Teva Neuroscience; has received funding for travel and/or speaker honoraria from Academic CME, Alcimed, Anthem Blue Cross, Genentech/Roche, Biogen IDEC, Novartis, CellGene, Seono, Epigene, Rocky Mountain MS Center, GLG Consulting, Ohio Health, TG Therapeutics, Topaz
Therapeutics, Dleara Lawyers, and Teva Neuroscience; and receives research support from Teva Neuroscience, Biogen, Actelion, Roche/Genentech, UT Southwestern, F. HoffmanLa Roche, TG Therapeutics, Inc., NIH/NINDS, and Rocky Mountain MS Center. J.A. Cohen has served on a scientific advisory board/as a consultant for Adamas, Celgene, Convelo, EMD Serono, Novartis, and PendoPharm; has received a speaker honorarium from Mylan; and serves as Co-Editor, Multiple Sclerosis Journal: Experimental, Translational and Clinical. E. Alvarez serves on scientific advisory boards for Biogen, Celgene, Genzyme, Genentech, Novartis, Teva, and TG Pharmaceuticals and receives research support from Acorda, Alkermes, Biogen, Genentech, Novartis, and Rocky Mountain MS Center. C.M. Hersh serves on scientific advisory boards for Biogen Idec, TEVA, and Genentech; has received speaker honoraria from TEVA, Genzyme, Novartis, and Biogen; serves on speakers' bureaus for TEVA, Genzyme, Novartis, and Biogen; and receives research support from Biogen and Genentech. Full disclosure form information provided by the authors is available with the full text of this article at Neurology.org/cp.

Received February 21, 2018. Accepted in final form May 8, 2018.

\section{References}

1. Gold R, Kappos L, Arnold DL, et al. Placebo-controlled phase 3 study of oral BG-12 for relapsing multiple sclerosis. N Engl J Med 2012;367:1098-1107.

2. Fox RJ, Miller DH, Phillips JT, et al. Placebo-controlled phase 3 study of oral BG-12 or glatiramer in multiple sclerosis. N Engl J Med 2012;367:1087-1097.

3. Brinkmann V, Davis MD, Heise CE, et al. The immune modulator FTY720 targets sphingosine 1-phosphate receptors. J Biol Chem 2002;277:21453-21457.

4. Kappos L, Radue E-W, O'Connor P, et al. A placebo-controlled trial of oral fingolimod in relapsing multiple sclerosis. N Engl J Med 2010;362:387-401.

5. Cohen JA, Barkhof F, Comi G, et al. Oral fingolimod or intramuscular interferon for relapsing multiple sclerosis. N Engl J Med 2010;362:402-415.

6. Paolicelli D, Manni A, Direnzo V, et al. Long-term cardiac safety and tolerability of fingolimod in multiple sclerosis: a postmarketing study. J Clin Pharmacol 2015;55: 1131-1136.

7. Baharnoori M, Lyons J, Dastagir A, Koralnik I, Stankiewicz JM. Nonfatal PML in a patient with multiple sclerosis treated with dimethyl fumarate. Neurol Neuroimmunol Neuroinflamm 2016;3:e274.

8. Lehmann-Horn K, Penkert H, Grein P, et al. PML during dimethyl fumarate treatment of multiple sclerosis: how does lymphopenia matter? Neurology 2016;87: 440-441.

9. Rosenkranz T, Novas M, Terborg C. PML in a patient with lymphocytopenia treated with dimethyl fumarate. N Engl J Med 2015;372:1476-1478.

10. FDA Drug Safety Communication. FDA warns about cases of rare brain infection with MS drug Gilenya (fingolimod) in two patients with no prior exposure to immunosuppressant drugs [online]. Available at: fda.gov/Drugs/DrugSafety/ucm 456919 htm. Accessed February 7, 2018.

11. Vollmer B, Nair KV, Sillau SH, Corboy J, Vollmer T, Alvarez E. Comparison of fingolimod and dimethyl fumarate in the treatment of multiple sclerosis: two-year experience. Mult Scler J Exp Transl Clin 2017;3:2055217317725102.

12. Hersh CM, Love TE, Bandyopadhyay A, et al. Comparative efficacy and discontinuation of dimethyl fumarate and fingolimod in clinical practice at 24-month follow-up. Mult Scler J Exp Transl Clin 2017;3:2055217317715485.

13. Wicks P, Rasouliyan L, Katic B, Nafees B, Flood E, Sasané R. The real-world patient experience of fingolimod and dimethyl fumarate for multiple sclerosis. BMC Res Notes 2016;9:434

14. Rosenbaum R, Rubin DB. The central role of the propensity score in observational studies for causal effects. Biometrika 1983;70:41-55.

15. Team RDC. R: a language and environment for statistical computing. Vienna: The $\mathrm{R}$ Foundation for Statistical Computing; 2014.

16. Hersh CM, Love TE, Cohn S, et al. Comparative efficacy and discontinuation of dimethyl fumarate and fingolimod in clinical practice at 12-month follow-up. Mult Scler Relat Disord 2016;10:44-52.

17. Rubin DB. Using propensity scores to help design observational studies: application to the tobacco litigation. Health Serv Outcomes Res Methodol 2001;2: 169-188.

18. Fox RJ, Chan A, Zhang A, et al. Comparative effectiveness using a matching-adjusted indirect comparison between delayed-release dimethyl fumarate and fingolimod for the treatment of multiple sclerosis. Curr Med Res Opin 2017;33:175-183.

19. Boster A, Nicholas J, Wu N, et al. Comparative effectiveness research of diseasemodifying therapies for the management of multiple sclerosis: analysis of a large health insurance claims database. Neurol Ther 2017;6:91-102. 

progressive course of multiple sclerosis. Brain 2013;136:3609-3617.

21. Manouchehrinia A, Westerlind H, Kingwell E, et al. Age related multiple sclerosis severity score: disability ranked by age. Mult Scler J 2017;23:1938-1946.

22. Giovannoni G, Turner B, Gnanapavan S, Offiah C, Schmierer K, Marta M. Is it time to target no evident disease activity (NEDA) in multiple sclerosis? Mult Scler Relat Disord 2015;4:329-333.

23. Nixon R, Bergvall N, Tomic D, Sfikas N, Cutter G, Giovannoni G. No evidence of disease activity: indirect comparisons of oral therapies for the treatment of relapsing-remitting multiple sclerosis. Adv Ther 2014;31:1134-1154.

24. Bar-Or A, Gold R, Kappos L, et al. Clinical efficacy of BG-12 (dimethyl fumarate) in patients with relapsing-remitting multiple sclerosis: subgroup analyses of the DEFINE study. J Neurol 2013;260:2297-2305.
25. Hutchinson M, Fox RJ, Miller DH, et al. Clinical efficacy of BG-12 (dimethyl fumarate) in patients with relapsing-remitting multiple sclerosis: subgroup analyses of the CONFIRM study. J Neurol 2013;260:2286-2296.

26. Derfuss T, Ontaneda D, Nicholas J, Meng X, Hawker K. Relapse rates in patients with multiple sclerosis treated with fingolimod: subgroup analyses of pooled data from three phase 3 trials. Mult Scler Relat Disord 2016;8: 124-130.

27. Kappos L, Gold R, Miller DH, et al. Effect of BG-12 on contrast-enhanced lesions in patients with relapsing-remitting multiple sclerosis: subgroup analyses from the phase 2b study. Mult Scler J 2012;18:314-321.

28. Meyniel C, Spelman T, Jokubaitis VG, et al. Country, sex, EDSS change and therapy choice independently predict treatment discontinuation in multiple sclerosis and clinically isolated syndrome. PLOS One 2012;7:e38661.

\section{Subspecialty Alerts by E-mail!}

Customize your online journal experience by signing up for e-mail alerts related to your subspecialty or area of interest. Access this free service by clicking on the "My Alerts" link on the home page. An extensive list of subspecialties, methods, and study design choices will be available for you to choose from-allowing you priority alerts to cutting-edge research in your field! 


\section{Neurology ${ }^{\circ}$ Clinical Practice}

\section{Discontinuation and comparative effectiveness of dimethyl fumarate and fingolimod in 2 centers}

Brandi Vollmer, Daniel Ontaneda, Anasua Bandyopadhyay, et al.

Neurol Clin Pract 2018;8;292-301 Published Online before print July 11, 2018

DOI 10.1212/CPJ.0000000000000487

This information is current as of July 11, 2018

$\begin{array}{ll}\begin{array}{l}\text { Updated Information \& } \\ \text { Services }\end{array} & \begin{array}{l}\text { including high resolution figures, can be found at: } \\ \text { http://cp.neurology.org/content/8/4/292.full.html }\end{array} \\ \text { References } & \text { This article cites } 25 \text { articles, } 1 \text { of which you can access for free at: } \\ \text { http://cp.neurology.org/content/8/4/292.full.html\#\#ref-list-1 } & \text { This article has been cited by } 4 \text { HighWire-hosted articles: } \\ \text { http://cp.neurology.org/content/8/4/292.full.html\#\#therarticles } & \text { This article, along with others on similar topics, appears in the } \\ \text { fitations } & \text { Mullowing collection(s): } \\ \text { Mubspecialty Collections } & \text { http://cp.neurology.org//cgi/collection/multiple_sclerosis } \\ & \begin{array}{l}\text { Information about reproducing this article in parts (figures,tables) or in } \\ \text { its entirety can be found online at: } \\ \text { http://cp.neurology.org/misc/about.xhtml\#permissions }\end{array} \\ \text { Permissions \& Licensing } & \begin{array}{l}\text { Information about ordering reprints can be found online: } \\ \text { http://cp.neurology.org/misc/addir.xhtml\#reprintsus }\end{array} \\ \text { Reprints } & \end{array}$

Neurol Clin Pract is an official journal of the American Academy of Neurology. Published continuously since 2011, it is now a bimonthly with 6 issues per year. Copyright Copyright $@ 2018$ The Author(s). Published by Wolters Kluwer Health, Inc. on behalf of the American Academy of Neurology.. All rights reserved. Print ISSN: 2163-0402. Online ISSN: 2163-0933.

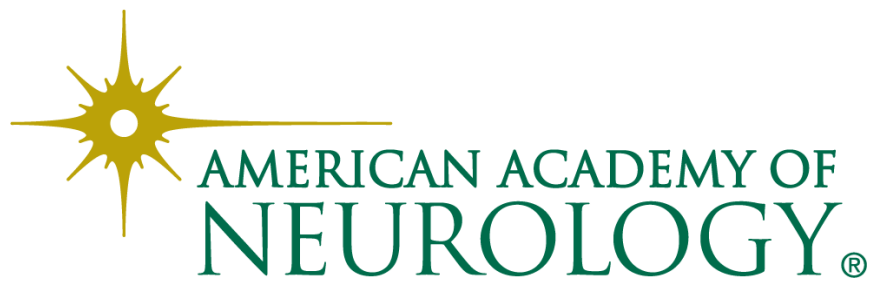

